\title{
Avaliação da sensibilidade ao contraste e da estereopsia em pacientes com lente intra-ocular multifocal
}

\author{
Contrast sensitivity and stereopsis in pseudophakic patients with multifocal \\ intraocularlens
}

\author{
Filipe de Oliveira ${ }^{1}$ \\ Cristina Muccioli ${ }^{2}$ \\ Luci Meire Pereira da Silva ${ }^{3}$ \\ Eduardo Sone Soriano ${ }^{4}$ \\ Carlos Eduardo Borges Souza ${ }^{5}$ \\ Rubens Belfort $\mathbf{J r}^{6}{ }^{6}$
}

\footnotetext{
Trabalho realizado no Departamento de Oftalmologia da Universidade Federal de São Paulo (UNIFESP).

${ }^{1}$ Tecnólogo Oftálmico do Setor de Pesquisa Clínica do Departamento de Oftalmologia e Preceptor do Curso de Graduação de Tecnologia Oftálmica da Universidade Federal de São Paulo (UNIFESP). São Paulo (SP).

2 Professora Adjunta Livre-docente e Chefe do Setor de Pesquisa Clínica do Departamento de Oftalmologia da UNIFESP. São Paulo (SP)

${ }^{3}$ Coordenadora de Pesquisa Clínica e MBA em Saúde do Departamento de Oftalmologia da UNIFESP. São Paulo (SP).

${ }^{4}$ Chefe do Instituto da Catarata (INCAT) do Departamento de Oftalmologia da UNIFESP. São Paulo (SP)

Pós-Graduando, nível doutorado, do Departamento de Oftalmologia da UNIFESP. São Paulo (SP).

${ }^{6}$ Professor Titular do Departamento de Oftalmologia da UNIFESP. São Paulo (SP)

Endereço para correspondência: Filipe de Oliveira Rua Botucatu, 822 - São Paulo (SP) CEP 04023-062 E-mail: filipe@oftalmo.epm.br

Recebido para publicação em 22.10.2004

Aprovação em 04.03.2005
}

\begin{tabular}{|c|}
\hline RESUMO \\
\hline 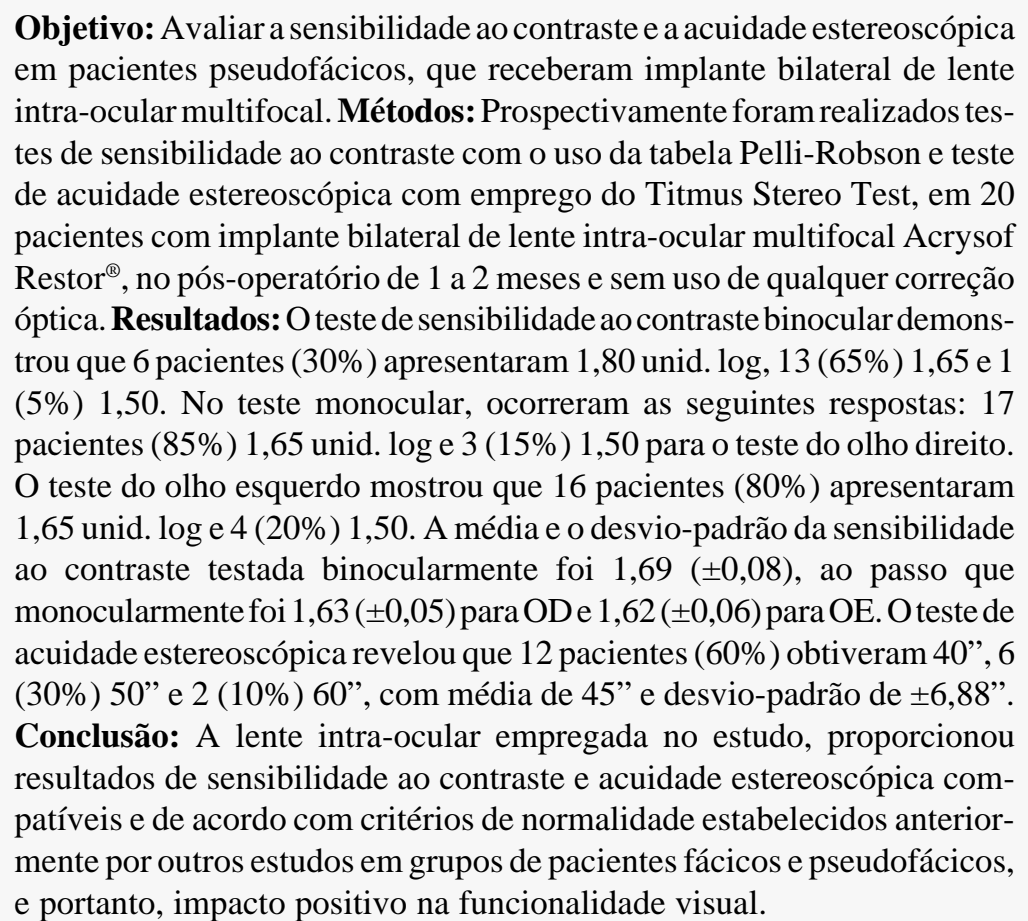 \\
\hline
\end{tabular}

Descritores: Sensibilidades de contraste; Percepção de profundidade; Pseudofacia, Implante de lente intra-ocular, Lentes intra-oculares; Catarata

\section{INTRODUÇÃO}

A acuidade visual (AV) refere-se à habilidade do sistema visual em identificar o menor optotipo em condição de máximo contraste, porém as imagens do mundo real do nosso cotidiano nem sempre se encontram nessa condição. Os objetos a nossa volta apresentam-se em diversos tamanhos e sob ampla variedade de contraste; tais como ocorrem ao amanhecer, entardecer, dias nublados ou sob nevoeiro, entre outras.

A visão humana se adapta a padrões de contraste por mudanças que ocorrem na sua sensibilidade perceptiva. O brilho de um objeto depende do seu contexto espacial, interpretação de cenas e de tridimensionalidade, sombras e outros níveis superiores de percepção de um objeto ${ }^{(1)}$. A detecção de gradientes de luminância, convencionalmente conhecida como sensibilidade ao contraste (SC), estabelece a base fundamental do processamento da visão ${ }^{(2)}$. 
A acuidade estereoscópica (AE) ou habilidade na percepção de profundidade ou tridimensionalidade baseia-se na estimulação simultânea e binocular de elementos retinianos discretamente díspares, e é derivada da separação horizontal entre os olhos ${ }^{(3)}$. Alguns estudos demonstram que os fatores que possivelmente estariam relacionados à estereopsia em pacientes afácicos e pseudofácicos são: idade; diferença entre os olhos na acuidade visual, equivalente esférico, astigmatismo e comprimento axial; aniseicônia; diâmetro pupilar e descentração da lente intra-ocular (LIO) $)^{(4)}$.

Diferentemente das LIOs monofocais convencionais, que normalmente corrigem apenas a visão para longe fazendo com que muitos pacientes necessitem de óculos para a correção da presbiopia pseudofácica ${ }^{(5)}$, as LIOs multifocais foram criadas para reduzir a dependência do uso de óculos para longe e para perto após a cirurgia de catarata e vêm ganhando aceitação como uma opção cirúrgica também com potencial refrativo ${ }^{(6)}$. A vantagem da LIO multifocal é que ela oferece ao cérebro a habilidade natural de adaptar-se à visão para longe e para perto, de acordo com a localização espacial do objeto da atenção visual. Essas LIOs têm a capacidade de projetar no plano retiniano um conjunto de imagens de variadas distâncias. O paciente pode beneficiar-se desta característica após se acostumar com a mesma, o que envolve um complexo processo cortical de elaboração e seleção ${ }^{(7)}$.

O desenvolvimento deste estudo, teve como objetivo demonstrar a metodologia e os resultados da $\mathrm{SC}$ e da $\mathrm{AE}$ em pacientes pseudofácicos que receberam implante bilateral de LIO multifocal, e verificar assim, a influência óptica desse modelo de LIO nesses resultados.

\section{MÉTODOS}

Um estudo prospectivo foi realizado no Departamento de Oftalmologia da UNIFESP e envolveu 20 pacientes com média de idade de 68,9 (variando de 50 a 78 anos), que foram incluídos num protocolo clínico investigacional para avaliar a eficácia de uma LIO multifocal com capacidade para corrigir a visão para longe e para perto após cirurgia da catarata.

Os pacientes foram submetidos à técnica cirúrgica de facoemulsificação com implante de LIO (Acrysof Restor ${ }^{\circledR}$ Alcon) bilateralmente e de forma não simultânea (o segundo implante ocorreu entre 2 a 3 semanas após o primeiro).

A SC monocular e binocular e a AE foram avaliadas no pós-operatório de 1 a 2 meses sempre pelo mesmo tecnólogo oftálmico (F.O).

O teste de SC foi realizado usando-se a tabela Pelli-Robson (Clement Clarke International, London) (Figura 1), que é composta por duas lâminas (uma para cada olho, para evitar memorização do paciente) e constituída de oito linhas de optotipos. Cada linha é composta por seis letras de mesmo tamanho $(4,9 \mathrm{x}$ $4,9 \mathrm{~cm})$, sendo que as três primeiras letras do lado esquerdo de cada linha, apresentam contraste maior que as três do lado direito. A redução de contraste ocorre a cada letra com acrés-

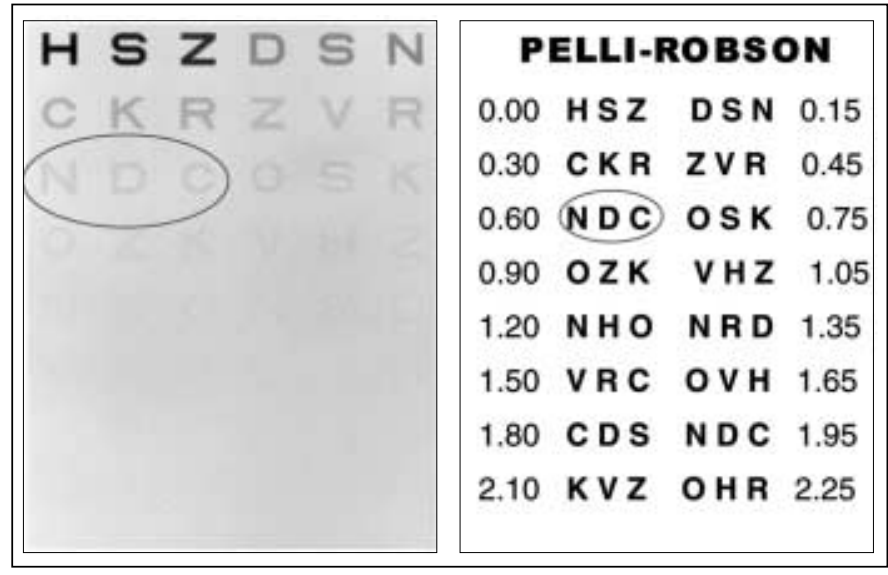

Figura 1 - Tabela de sensibilidade ao contraste Pelli-Robson. Os valores dos resultados do teste são os números de cada lado da tabela dados em unidade logarítmica (unid. log), e correspondem a cada grupo de três letras. No exemplo, o conjunto de letras circulado indica um contraste de $25 \%\left(1 / 10^{0,60}=0,25=25 \%\right)$

cimo de 0,05 unidade logarítmica (unid. log), e portanto quantificada a cada grupo de três letras em 0,15 unid. log. Esses valores são representados como sensibilidade de contraste logarítmica e correspondem à recíproca do contraste (sensibilidade ao contraste $=1 /$ contraste) . A primeira letra da primeira linha tem um contraste de $100 \%$ e a última letra da última linha tem $0,6 \%{ }^{(8)}$. Conforme recomendações da tabela, o teste foi realizado numa sala com iluminação uniforme e a tabela apresentando uma luminância média de $85 \mathrm{~cd} / \mathrm{m}^{2}$, com uma faixa de variação aceitável entre 60 a $120 \mathrm{~cd} / \mathrm{m}^{2}$, testada previamente utilizando-se o fotômetro Gossen Starlite. O paciente sentado a 1 metro da tabela, proporcionou a visualização dos optotipos a uma freqüência espacial de cerca de 1 ciclo por grau (cpg) nessa distância de teste ${ }^{(9)}$. $\mathrm{O}$ valor da SC considerada, foi aquele correspondente ao último grupo de três letras onde o paciente pode ler corretamente pelo menos duas.

O Titmus Stereo Test (Stereo Optical Co., Inc., Chicago, IL) foi empregado posteriormente para o exame de estereopsia. Trata-se de um teste que é composto por um livro de duas faces, sendo que em cada face estão dispostas figuras que foram projetadas em duplicidade e com uma disparidade horizontal entre si. Com o uso de um óculos polarizado e o livro posicionado entre 30 e $40 \mathrm{~cm}$ dos olhos, o paciente foi orientado a indicar as figuras que ele percebeu em "relevo" (tridimensão). Essa percepção de tridimensionalidade é a disparidade de imagem, e é medida em segundos de arco ("). Quantitativamente a AE nesse teste varia numa faixa entre 3000" e 40", sendo que o nível dessa disparidade vai diminuindo progressivamente à medida que o paciente é capaz de identificá-las. Portanto, quanto menor o valor numérico em segundos de arco, maior é a acuidade estereoscópica (Figura 2).

Ambos os testes foram realizados sem o emprego de qualquer tipo de correção óptica devido ao fato da LIO implantada ser designada para a correção da visão para longe e para perto sem a dependência do uso de óculos. 


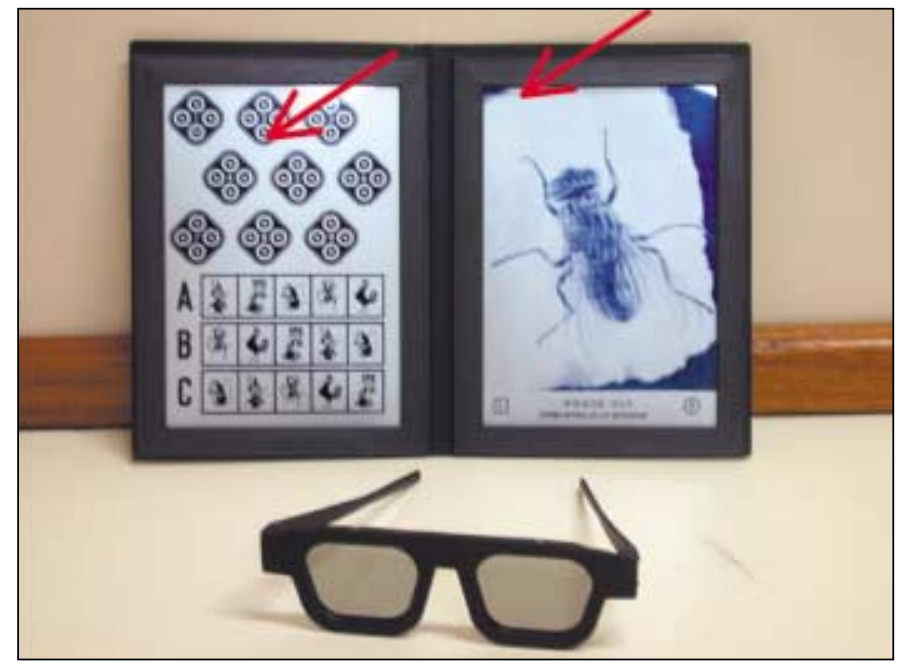

Figura 2 - Titmus Stereo Test: óculos com lentes polarizadas e livro com figuras. As setas indicam a mosca (corresponde a uma estereopsia grosseira = $3000 "$ ") e o último conjunto de círculos numerados ( corresponde a uma estereopsia refinada $=\mathbf{4 0}$ ”)

Os dados referentes à $\mathrm{AV}$ para longe e para perto, sem uso de correção óptica, estão apresentados na tabela 2. Os pacientes apresentavam um diâmetro pupilar que variava de 4,0 e $5,0 \mathrm{~mm}$, que fora obtido com régua milimetrada antes dos testes e sob as condições de luminosidade da sala.

Os pacientes envolvidos nesse estudo, assinaram um termo de consentimento livre e esclarecido previamente aprovado pelo Comitê de Ética em Pesquisa da Universidade Federal de São Paulo (UNIFESP), de acordo com as diretrizes da Declaração de Helsinki ${ }^{(10)}$.

\section{RESULTADOS}

O teste de SC demonstrou que: 6 pacientes (30\%) apresentaram 1,80 unid. log, 13 (65\%) 1,65 unid. $\log$ e 1 (5\%) 1,50 unid. $\log$. No teste monocular, ocorreram as seguintes respostas: 17 pacientes $(85 \%)$ 1,65 unid. log e $3(15 \%)$ 1,50 para o teste do olho direito (OD). $\mathrm{O}$ teste do olho esquerdo (OE) mostrou que 16 pacientes (80\%) apresentaram 1,65 unid. log e $4(20 \%)$ 1,50. A média e o desvio-padrão da sensibilidade ao contraste testada monocular e binocularmente foram: $1,63( \pm 0,05)$ para OD, 1,62 $( \pm 0,06)$ para OE e $1,69( \pm 0,08)$ para o exame binocular (Tabela 1).

O teste de AE revelou que 12 pacientes (60\%) obtiveram 40 ", $6(30 \%)$ 50" e 2 (10\%) 60", com média de 45" e desviopadrão de \pm 6.88 " (Gráfico 1$)$.

\section{DISCUSSÃO}

Atualmente a investigação da SC e da AE em pacientes pseudofácicos, tem se tornado freqüente devido ao fato destes exames fornecerem informações muito próximas da qualidade visual do dia-a-dia desses pacientes. Porém, alguns es-

\begin{tabular}{|lcccc|}
\hline \multicolumn{5}{|c|}{ Tabela 1. Distribuição de escores de sensibilidade ao contraste } \\
monocular e binocular $(\mathbf{N}=\mathbf{2 0})$
\end{tabular}

Tabela 2. Acuidade visual para longe e para perto sem uso de correção óptica

$\begin{array}{lr}\text { AV (longe) } & \mathbf{N}(\%) \\ 20 / 20 \text { ou melhor } & 16(80) \\ 20 / 32 \text { a } 20 / 25 & 4(20) \\ \text { AV (perto) } & \mathbf{N}(\%) \\ 20 / 20 \text { ou melhor } & 17(85) \\ 20 / 32 \text { a } 20 / 25 & 3(15)\end{array}$

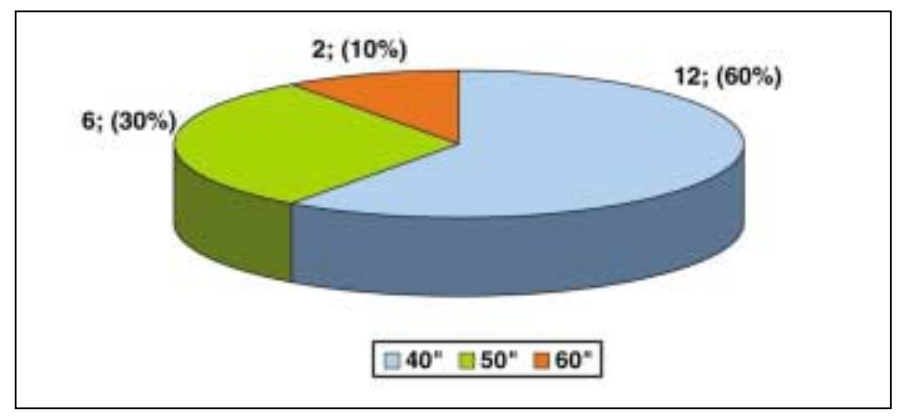

Gráfico 1 - Acuidade estereoscópica / Titmus Stereo Test

tudos envolvendo LIOs multifocais, mostram resultados de SC consideravelmente diferentes ${ }^{(11-18)}$; entretanto deve-se ter cuidado com comparações, devido às diferenças entre pacientes, tabelas, condições de iluminação e examinadores.

A avaliação da SC pode ser realizada com padrões de grade ("gratings") ou com optotipos. A SC nos testes com padrões de grade é definida em diversas freqüências espaciais (tamanho do alvo), usualmente entre 1,5 e $18,0 \mathrm{cpg}$. O teste com optotipos, como a tabela Pelli-Robson, mede a $\mathrm{SC}$ a baixas freqüências (cerca de $1 \mathrm{cpg}$ ) na distância de teste em que é recomendada (1 m), sendo que outras frequiências podem ser pesquisadas desde que o examinador mude essa distância. Mäntyjärvi e Laitinem, num estudo recente não encontraram diferenças estatisticamente significantes nos resultados dos testes a 1 e $3 \mathrm{~m}$ realizados binocularmente em diversas faixas etárias ${ }^{(9)}$.

As instruções da tabela Pelli-Robson, sugerem que se a correção para longe for necessária, pode-se adicionar +0,75 D no teste realizado a $1 \mathrm{~m}$, entretanto isso não ocorreu nesse estudo, descartando assim qualquer influência óptica que não fosse inerente à LIO e condizendo assim, com a metodologia de um trabalho recente desenvolvido na Finlândia, sobre índices de normalidade para SC em variadas faixas etárias ${ }^{(9)}$.

A análise gráfica dos resultados de SC em diversas freqüências espaciais, assemelha-se a uma parábola; o pico da 
SC ocorre nas freqüências espaciais médias, contrariamente às freqüências baixas e altas que constituem menores valores de $\mathrm{SC}$ (Gráfico 2).

A investigação da SC com tabelas de optotipos tem sido realizada em estudos com pacientes fácicos e pseudofáci$\cos ^{(19-20)}$, podendo o cirurgião decidir a partir dos resultados de SC, qual olho será operado primeiro em casos de olhos com mesma AV. Apesar de atualmente existirem outros métodos para avaliação da SC, o presente estudo usou a tabela PelliRobson, devido à análise da SC a baixas freqüências espaciais sugerir alguma relação com dispersão ou borramento na percepção da luz ${ }^{(21)}$. Isso é particularmente importante, devido ao fato da luz que atravessa a LIO multifocal ser dividida em múltiplos feixes que se projetam no plano retiniano. Além disso, o fato dos optotipos serem letras, torna o exame mais rápido e seguro ${ }^{(22)}$ e parece ser um melhor indicador da percepção do mundo real desses pacientes ${ }^{(23)}$. Outro aspecto importante, é que a percepção de optotipos exige habilidades complexas do sistema visual se comparada à de "gratings", pois envolve um conjunto de informações hierarquizadas de detecção, discriminação e identificação ${ }^{(24)}$.

Apesar de Rubin et al., terem encontrado escores de 1,50 $( \pm 0,08)$ em olhos com implante de LIO multifocal e $1,65( \pm 0,14)$ com LIO monofocal, o estudo atual revelou um desempenho superior da LIO multifocal na detecção da SC $[1,69( \pm 0,08)]^{(25)}$.

Num estudo envolvendo pacientes normais, Elliott e Whitaker indicaram valores de normalidade com uso da tabela Pelli-Robson, de 1,65 para a faixa etária de 20 a 50 anos e de 1,50 para indivíduos com mais de 50 anos de idade, demonstrando assim que os resultados do presente estudo são discretamente melhores $^{(26)}$. Diferentemente de um estudo com pacientes fácicos normais, com idade superior a 60 anos, onde se verificou um escore médio de 1,90 unid. $\log ^{(9)}$.

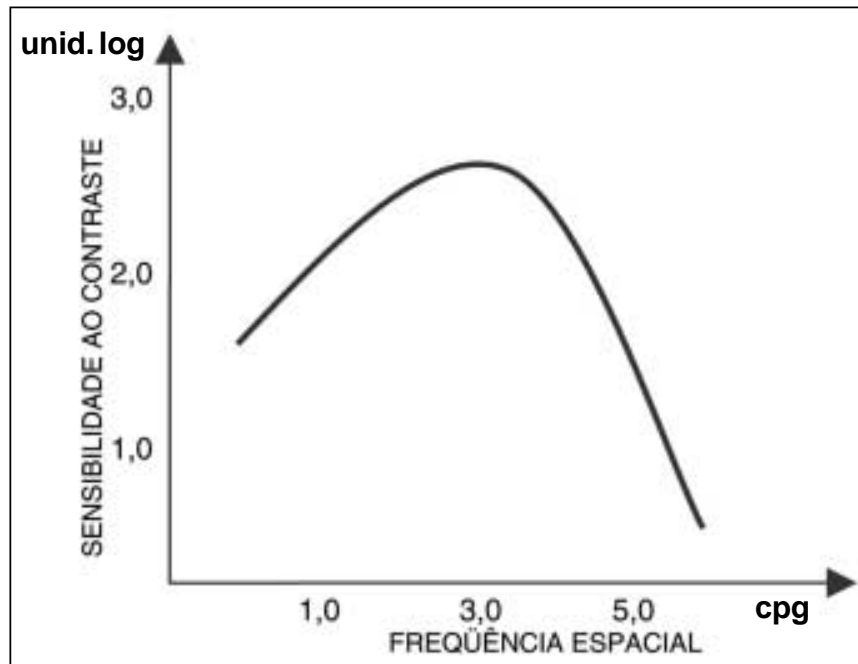

unid. $\log =$ unidades logarítmicas; $\mathrm{cpg}=$ ciclos por grau

Gráfico 2 - Curva característica da sensibilidade ao contraste sob baixas, médias e altas freqüências espaciais em pacientes normais
A influência do diâmetro pupilar na SC pode ser desconsiderada nesse estudo, conforme verificado anteriormente por Woodhouse que não encontrou alterações significativas na SC em pacientes com diâmetro pupilar entre 2 e $6 \mathrm{~mm}^{(27)}$.

Embora diversos estudos com LIO multifocal abordem a influência da formação da imagem no plano retiniano e sua interferência na $\mathrm{SC}$, poucos avaliam essa interferência na $\mathrm{AE}^{(28)}$. A propriedade óptica desse modelo de LIO, pode ser avaliada pelo nível da $\mathrm{AE}$, uma vez que dois critérios devem ser respeitados para que ocorra corticalmente o processamento da estereopsia: 1) as imagens provenientes de cada olho devem ter forma, tamanho, cor e brilho mais próximas possíveis e 2) essas imagens devem estimular a mesma região cerebral, portanto a retina e os elementos que dela se originam devem estar íntegros.

Alguns autores ${ }^{(29)}$ num estudo de estereopsia em pacientes pseudofácios (média de idade de 69,66 $\pm 12,25$ anos) com LIO monofocal, verificou que aproximadamente $71 \%$ dos pacientes apresentaram acuidade estereoscópica máxima no Titmus Test (40") e os demais $19 \%$ entre 50" e 800 "; o presente estudo revelou $60 \%$ dos pacientes com o mesmo resultado e $40 \%$ entre 50 " e 60 ", demonstrando uma faixa de variação e média menores e portanto clinicamente melhores. Num estudo da função binocular em pacientes com LIO multifocal, Shoji e Shimizu encontraram $84,6 \%$ dos pacientes com implante bilateral com estereopsia de 60" ou melhor e um paciente com 140"; no grupo de pacientes com implante unilateral, $42,8 \%$ apresentavam 60 " ou melhor e $28,6 \%$ com 140 ". Ainda revelaram, nesse estudo, que nos pacientes com implante unilateral, a estereopsia parecia depender da acomodação; sendo que indícios de catarata ou presbiopia no olho contralateral foram os fatores que ocasionaram redução da habilidade estereoscópica ${ }^{(30)}$.

\section{CONCLUSÃO}

A LIO multifocal empregada nesse estudo, produziu resultados de SC e de AE compatíveis e de acordo com critérios de normalidade estabelecidos anteriormente por outros autores em grupos de pacientes fácicos e pseudofácicos, demonstrando assim, sua eficiência óptica, e portanto impacto positivo na funcionalidade visual.

\section{AGRADECIMENTOS}

À Prof ${ }^{\mathrm{a}}$. Dr ${ }^{\mathrm{a}}$. Sueli de Faria Müller, do Departamento de Oftalmologia da UNIFESP, pela revisão bibliográfica e à Valéria Alves, coordenadora de estudos do Setor de Pesquisa Clínica em Oftalmologia (SEPECO), pela colaboração e apoio.

\section{ABSTRACT}

Purpose: To evaluate the contrast sensitivity and stereopsis tests in patients who underwent bilateral implantation of multifocal intraocular lens. Methods: Tests of contrast sensitivity using the Pelli-Robson chart and stereopsis evaluation with 
the Titmus Stereo Test were performed in 20 patients $30-60$ days after the bilateral implantation of Acrysof Restor ${ }^{\circledR}$ multifocal intraocular lens. Results: The binocular contrast sensitivity test demonstrated that 6 patients $(30 \%)$ presented 1.80 $\log$ units, $13(65 \%) 1.65$ and in only $1(5 \%)$ the sensitivity was $1.50 \log$ units. By the other hand, the monocular test in right eye showed 17 patients $(85 \%)$ with $1.65 \log$ units and $3(15 \%)$ with 1.50 . In the left eye, the test presented 16 patients $(80 \%)$ with $1.65 \log$ units and $4(20 \%)$ with $1.50 \log$ units. The average and the standard deviation of the contrast sensitivity were $1.63( \pm 0.05)$ for right eye, $1.62( \pm 0.06)$ for left eye and 1.69 $( \pm 0.08)$ for binocular test. Stereopsis test disclosed 12 patients (60\%) presenting 40", 6 (30\%) 50" and only 2 (10\%) 60" (average: 45 " and standard deviation: 6.88"). Conclusion: The Acrysof Restor ${ }^{\circledR}$ intraocular lens provided results of contrast sensitivity and stereopsis in accordance with the criteria of normality established previously in other studies for phakic and pseudophakic patients. Therefore, this intraocular lens does not decrease visual functionality.

Keywords: Contrast Sensitivity; Depth Perception; Pseudophakia; Lens Implantation, Intraocular; Lenses, Intraocular; Cataract

\section{REFERÊNCIAS}

1. Eagleman DM, Jacobson JE, Sejnowski TJ. Perceived luminance depends on temporal context. Nature. 2004;428(22):854-6.

2. Hawken MJ, Blakemore C, Morley JW. Development contrast sensitivity and temporal-frequency selectivity in primate lateral geniculate nucleus. Exp Brain Res. 1997;114(1):86-98.

3. Patterson R, Martin WL. Human stereopsis. Hum Factors. 1992;34(6):669-92.

4. Hayashi K, Hayashi H. Stereopsis in bilaterally pseudophakic patients. J Cataract Refract Surg. 2004;30(7):1466-70.

5. Oliveira F, Silva LMP, Muccioli C, Soriano ES, Freitas LL, Belfort Jr R. Qualidade de vida de pacientes pseudofácicos submetidos à cirurgia de catarata com implante de lente intra-ocular acomodativa. Arq Bras Oftalmol. 2004; 67(3):469-74

6. Montés-Micó R, Alió JL. Distance and near contrast sensitivity function after multifocal intraocular lens implantation. J Cataract Refract Surg. 2003; 29(4):703-11.

7. Ravalico G, Baccara F, Bellavitis A. Refractive bifocal intraocular lens and pupillary diameter. J Cataract Refract Surg. 1992;18(6):594-7.

8. Mäntyjärvi M, Tuppurainen K. Cataract in traffic. Graefe's Arch Clin Exp Ophthalmol. 1999;237(4):278-82.
9. Mäntyjärvi M, Laitinen T. Normal values for the Pelli-Robson contrast sensitivity test. J Cataract Refract Surg. 2000;27(2):261-6.

10. Declaration of Helsinki: recommendations guiding medical doctors in biomedical research involving human subjects. In: $41^{\circ}$ World Medical Assembly. Hong Kong: World Medical Association; 1989.

11. Schmitz S, Dick HB, Krummenauer F, Schwenn O, Krist R. Contrast sensitivity and glare disability by halogen light after monofocal and multifocal intraocular lens implantation. Br J Ophthalmol. 2000;84(10):1109-12.

12. Pieh S, Lackner B, Hanselmayer G, Zohrer R, Sticker M, Weghaup H, et al. Halo size under distance and near conditions in refractive multifocal intraocular lenses. Br J Ophthalmol. 2001;85(7):816-21.

13. Akutsu H, Legge GE, Showalter M, Lindstrom RL, Zabel RW, Kirby VM. Contrast sensitivity and reading through multifocal intraocular lenses. Arch Ophthalmol. 1992;110(8):1076-80.

14. Ravalico G, Baccara F, Rinaldi G. Contrast sensitivity in multifocal intraocular lenses. J Cataract Refract Surg. 1993;19(1):22-5.

15. Williamson W, Poirier L, Coulon P, Verin P. Compared optical performances of multifocal and monofocal intraocular lenses (contrast sensitivity and dynamic visual acuity). Br J Ophthalmol. 1994;78(4):249-51.

16. Pieh S, Weghaupt H, Skorpik C. Contrast sensitivity and glare disability with diffractive and refractive multifocal intraocular lenses. J Cataract Refract Surg. 1998;24(5):659-62.

17. Yang HC, Chung SK, Baek NH. Decentration, tilt and near vision of the array multifocal intraocular lens. J Cataract Refract Surg. 2000;26(4):586-9.

18. Hayashi K, Hayashi H, Nakao F, Hayashi F. Correlation between pupillary size and intraocular lens decentration and visual acuity of a zonal-progressive multifocal lens and a monofocal lens. Ophthalmology. 2001;108(11):2011-7.

19. Tan JC, Spalton DJ, Arden GB. Comparison of methods to assess visual impairment from glare and light scattering with posterior capsule opacification. J Cataract Refract Surg. 1998;24(12):1626-31.

20. Arens B, Freudenthaler N, Quentin CD. Binocular function after bilateral implantation of monofocal and refractive multifocal intraocular lenses. J Cataract Refract Surg. 1999;25(3):399-404.

21. Elliot DB. Evaluating visual function in cataract. Optom Vis Sci. 1993; 70(11):896-902.

22. Pelli DG, Robson JG. Are letters better than gratings? Clin Vision Sci. 1991;6(5)409-11.

23. Owsley C, Sloane ME. Contrast sensitivity, acuity and the perception of "real world" targets. Br J Ophthalmol. 1987;71(19):791-6.

24. Bradley A, Ohzawa D. A comparison of contrasts detection and discrimination. Vision Res. 1986;26(6):991-7.

25. Rubin GS, Adamsons IA, Stark WJ. Comparison of acuity, contrast sensitivity and disability glare before and after cataract surgery. Arch Ophthalmol. 1993;111(1):56-61.

26. Elliot DE, Whitaker D. Clinical contrast sensitivity chart evaluation. Ophthalmic Physiol Opt. 1992;12(3):275-80.

27. Woodhouse JM. The effect of pupil size on grating detection at various contrast levels. Vision Res. 1975;15(6):645-8.

28. Häring G, Gronemeyer A, Hedderich J, Decker W. Stereoacuity and aniseikonia after unilateral and bilateral implantation of the Array refractive multifocal intraocular lens. J Cataract Refract Surg. 1999;25(8):1151-6.

29. Katsumi O, Miyajima H, Ogawa T, Hirose T. Aniseikonia and stereoacuity in pseudophakic patients. Ophthalmology. 1992;99(8):1270-7.

30. Shoji N, Shimizu K. Binocular function of the patients with the refractive multifocal intraocular lens. J Cataract Refract Surg. 2002;28(6):1012-7.

\section{Ao enviar um artigo para publicação, leia ATENTAMENTE as instruções para autores, constante no final de cada fascículo.}

